\title{
Conductance fluctuations in mesoscopic normal-metal/superconductor samples
}

\author{
Klaus Hecker ${ }^{1}$, Helmut Hegger ${ }^{1}$, Alexander Altland ${ }^{2,3}$, and Klaus Fiegle ${ }^{4}$ \\ ${ }^{1}$ II. Physikalisches Institut, Universität zu Köln, D-50937 Köln, Germany \\ ${ }^{2}$ Institut für Theoretische Physik, Universität zu Köln, D-50937 Köln, Germany \\ 3 Cavendish Laboratory, Madingley Road, Cambridge CB3 OHE, UK \\ ${ }^{4}$ I. Physikalisches Institut, Universität zu Köln, D-50937 Köln, Germany
}

(February 7, 2018)

\begin{abstract}
We study the magnetoconductance fluctuations of mesoscopic normal-metal/superconductor (NS) samples consisting of a gold-wire in contact with a niobium film. The magnetic field strength is varied over a wide range, including values that are larger than the upper critical field $B_{c 2}$ of niobium. In agreement with recent theoretical predictions we find that in the NS sample the rms of the conductance fluctuations $(\mathrm{CF})$ is by a factor of $2.8 \pm 0.4$ larger than in the high field regime where the entire system is driven normal conducting. Further characteristics of the CF are discussed.
\end{abstract}

PACS numbers: 73.23.-b, 73.50.Jt, 74.80.-g

At low temperatures disordered metals smaller than the phase coherence length $L_{\varphi}$ (mesoscopic systems) exhibit a host of quantum fluctuation phenomena like e.g. universal fluctuations of the electrical conductance [1] 3 . Recently it has become clear that additional mechanisms of quantum coherence arise when a mesoscopic normal metal sample $(\mathrm{N})$ is brought in contact with a superconductor (S) 416]. These processes, which are caused by the interference of electrons and Andreev reflected holes [17], manifest themselves in the emergence of additional universality classes [th and an altered fluctuation behaviour of various mesoscopic observables. In particular the fluctuations of the magneto-conductance are still universal of order $e^{2} / h$ but tend to exceed the normalmetal fluctuations by numerical factors of order unity. These enhancement factors are a consequence of (i) the fact that two elementary charges are transferred per Andreev reflection [4, 5, 12, 13 and (ii) the presence of diffusionlike modes which are absent in the pure $\mathrm{N}$-case. To be specific, it has been found that in the presence of an external magnetic field the rms amplitude of the conductance fluctuations $\operatorname{rms}\left(G_{\mathrm{NS}}\right)$ exceeds its normal metal $\operatorname{rms}\left(G_{\mathrm{N}}\right)$ value by a factor of $2 \sqrt{2}$. This result holds true in the presence [4] or absence [4, [] of spin-orbit scattering.

The fluctuation characteristics of a NS sample are strongly affected by magnetic fields. At least three different regimes with qualitatively different behaviour can be identified [cf. Fig.1](a)]: (i) The presence of the proximity effect gives rise to a resistance minimum for small magnetic fields. In our samples the proximity effect is supressed by fields of order $B_{1} \sim 0.1 \mathrm{~T}$ [18]. (ii) For intermediate fields larger than $B_{1}$ but smaller than the upper critical field of the superconductor $B_{c 2}$, the proximity effect is supressed but the process of Andreev reflection is still active. (iii) For fields larger than $B_{c 2}$ the superconductivity is globally destroyed.

Most experiments of other groups were performed in regime (i) [7 11. In contrast, we have studied the magneto-CF in the regimes (ii) and (iii). We are thus in a position to compare the fluctuation behaviour of one and the same sample in both regimes NS and pure N. As a result, we find that the CF of the NS system are enhanced as compared to those of the $\mathrm{N}$ system. The ratio $\operatorname{rms}\left(G_{\mathrm{NS}}\right) / \operatorname{rms}\left(G_{\mathrm{N}}\right)$ turns out to be in good agreement with the theoretical prediction.

The inset of figure 11(a) shows the sample layout. The sample consists of a $\mathrm{Nb}$ contact, a mesoscopic Au wire and a second $\mathrm{Au}$ contact. This layout corresponds to a mesoscopic two-probe arrangement [19]. Far outside the coherence volume the two contacts are split-up to perform a macroscopic four-point measurement. The samples were prepared in two successive steps using electronbeam lithography and lift-off technique [20]. In a first step the $\mathrm{Nb}$ contact was prepared by in situ deposition of a $30 \mathrm{~nm} \mathrm{Nb}$ and a $10 \mathrm{~nm} \mathrm{Au}$ layer, which prevents oxidation of the $\mathrm{Nb}$. In a second process the $\mathrm{Au}$ wire of length $L \simeq 1 \mu \mathrm{m}$ (width $W \simeq 180 \mathrm{~nm}$, thickness $t \simeq 30$ $\mathrm{nm}$ ) and the $\mathrm{Au}$ contact was produced. Both the $\mathrm{Nb}$ and $\mathrm{Au}$ layers were dc magnetron sputtered. We report on measurements of two samples which had a normal state resistance $R_{\mathrm{N}} \sim 15 \Omega$ at low temperatures (see Table (I). From the residual resistance ratio and from the sheet resistance of wide $\mathrm{Nb}$ and $\mathrm{Au}$ films of the same thickness we derive elastic mean free paths of $\ell_{A u} \simeq 32 \mathrm{~nm}$, $\ell_{N b} \simeq 5 \mathrm{~nm}$ and diffusion constants of $D_{A u} \simeq 150 \mathrm{~cm}^{2} / \mathrm{s}$, $D_{N b} \simeq 23 \mathrm{~cm}^{2} / \mathrm{s}$. The Thouless energy $E_{T h}=h D_{A u} / L_{\varphi}^{2}$ for our systems is $E_{T h} \simeq 100 \mu \mathrm{eV}$ [21]. The critical temperature of the $\mathrm{Nb}$ is $T_{c} \simeq 8 \mathrm{~K}$, for $\mathrm{Nb}$ films with and without a $\mathrm{Au}$ layer, and $B_{c 2}(T=100 \mathrm{mK}) \simeq 2.5 \mathrm{~T}$. The measurements were performed in a ${ }^{3} \mathrm{He}-{ }^{4} \mathrm{He}$-dilution refrigerator at temperatures down to $T_{\text {min }} \simeq 45 \mathrm{mK}$ [19]. The conductance $G(B)$ was measured by means of a standard lock-in technique for magnetic fields up to $B=9$ $\mathrm{T}$. We used small measurement currents $I_{a c}=1-3 \mu \mathrm{A}$ at temperatures below $1 \mathrm{~K}$. Larger currents were used at $T>1 \mathrm{~K}$ always under the condition $V_{a c}<k_{B} T / e$.

We now turn to the discussion of our results. Figure 1 (b) shows the conductance fluctuations for both the NS- and the N-case as a function of the magnetic field. 
TABLE I. $R_{\mathrm{N}}$ : normal state resistance at $T=50 \mathrm{mK}$ and $B=4 \mathrm{~T}, R_{\mathrm{NS}}$ : resistance in the intermediate regime (ii) at $T=50 \mathrm{mK}$ and $B=1 \mathrm{~T}$ (see Fig.11).

\begin{tabular}{lcc}
\hline \hline & sample 1 & sample 2 \\
\hline$R_{\mathrm{N}}(\Omega)$ & 15.87 & 14.34 \\
$R_{\mathrm{NS}}(\Omega)$ & 11.60 & 9.72 \\
$\operatorname{rms}\left(G_{\mathrm{NS}}\right)\left(e^{2} / h\right)$ & $0.16 \pm 0.02$ & $0.14 \pm 0.02$ \\
$\operatorname{rms}\left(G_{\mathrm{N}}\right)\left(e^{2} / h\right)$ & $0.058 \pm 0.003$ & $0.050 \pm 0.004$ \\
$\operatorname{rms}\left(G_{\mathrm{NS}}\right) / \operatorname{rms}\left(G_{\mathrm{N}}\right)$ & $2.8 \pm 0.4$ & $2.8 \pm 0.4$ \\
\hline \hline
\end{tabular}

Since the magnetoconductance is measured in a mesoscopic two-probe configuration, its fluctuations are symmetric with respect to a reversal of the magnetic field [19,22. For this reason only the part $B>0 \mathrm{~T}$ of the magnetofingerprints $\Delta G(B)$ is shown. The NS-CF at low magnetic fields are clearly larger than in the $\mathrm{N}$-case $(B \geq 3.1 \mathrm{~T})$.

We obtain values of $\operatorname{rms}\left(G_{\mathrm{NS} 1}\right)=0.16 \pm 0.02 e^{2} / h$ and $\operatorname{rms}\left(G_{\mathrm{N} 1}\right)=0.058 \pm 0.003 e^{2} / h$ for sample 1 (see table I and Figs. 1 3 ). For sample 2 the rms values are slightly smaller: $\operatorname{rms}\left(G_{\mathrm{NS} 2}\right)=0.14 \pm 0.02 e^{2} / h$ and $\operatorname{rms}\left(G_{\mathrm{N} 2}\right)=$ $0.050 \pm 0.004 e^{2} / h$ (see table If and Fig.3). Since the magnetic-field range for the calculation of the NS-CF is much smaller $\left(\Delta B_{\mathrm{NS}}=1.5 \mathrm{~T}\right)$ than the range of the $\mathrm{N}$ $\mathrm{CF}\left(\Delta B_{\mathrm{N}}=5.9 \mathrm{~T}\right)$ the uncertainty for the $\operatorname{rms}\left(G_{\mathrm{NS}}\right)$ values is substantially larger.

We note that the measured values of the CF are much smaller than the results obtained in zero temperature theoretical calculations. To understand better the origin of this discrepancy, viz. the combined effect of finite temperatures and dephasing effects, let us briefly recall a few known theoretical predictions about the CF of diffusive mesoscopic systems. As has been shown diagrammatically (cf. [2] and references therein) the CF of quasione-dimensional wires in the presence of both spin-orbit interactions and a magnetic field are given by

$$
\begin{aligned}
& \left.\begin{array}{l}
\operatorname{rms}\left(G_{\mathrm{N}}\right)^{2} \\
\operatorname{rms}\left(G_{\mathrm{NS}}\right)^{2}
\end{array}\right\}=\frac{6 e^{4}}{h^{2}}\left\langle\sum_{q}\left(\frac{h D / L_{\mathrm{eff}}^{2}}{h D q^{2}+h / \tau_{\varphi}+i \epsilon}\right)^{2}\right\rangle_{\epsilon} \times \\
& \times\left\{\begin{array}{l}
1 \\
8
\end{array}\right. \text {. }
\end{aligned}
$$

Here $\langle\ldots\rangle_{\epsilon}:=\frac{1}{2 k_{B} T} \int_{-k_{B} T}^{k_{B} T} d \epsilon(\ldots)$ stands for a temperature induced energy averaging procedure [2], $\sum_{q}$ denotes a summation over quantized momenta $q=$ $\pi / L_{\text {eff }}, 2 \pi / L_{\text {eff }}, \ldots$ and it has been assumed that the wire's cross section $L_{\perp} \ll L_{\text {eff }}$ (i.e. $h D / L_{\perp}^{2}$ is by far larger than any relevant energy scale in the problem which means that the wire can be regarded as 'quasi'one-dimensional [2]). The phenomenological parameter $\tau_{\varphi}\left(L_{\varphi}=\sqrt{D \tau_{\varphi}}\right)$ accounts for the various dephasing mechanisms which lead to a destruction of the conductance fluctutions. Whereas the formula for the Ncase can be found at various places in the literature (cf. e.g. 23] for a reference with correct prefactors) the NSformula is less standard and deserves a few comments.
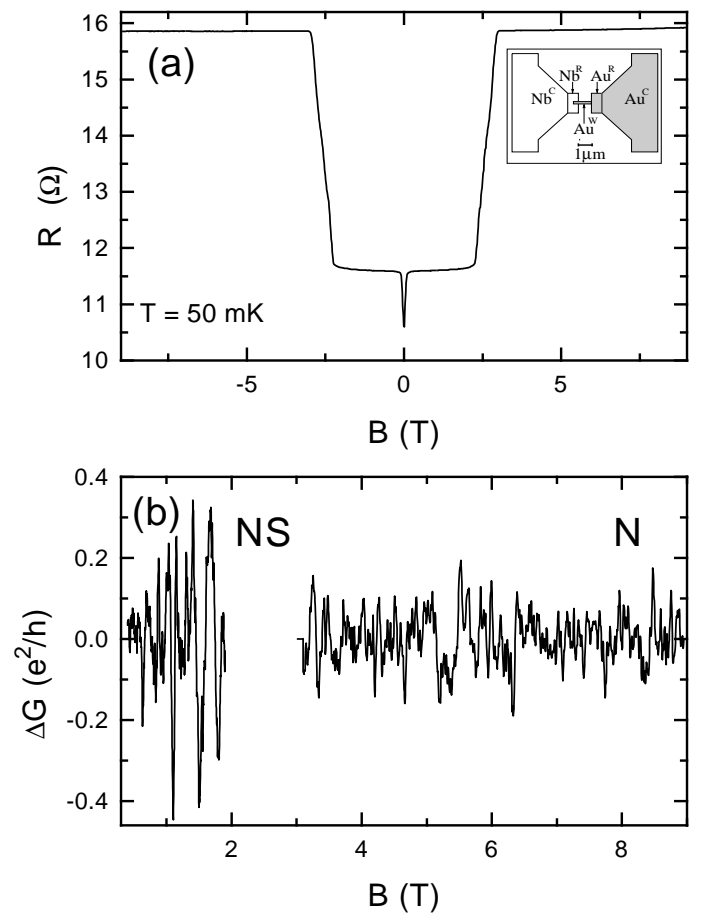

FIG. 1. (a) Magnetoresistance of sample 1. Three regimes can be identified (i) For small magnetic fields $\left(B_{1} \simeq 0.1 \mathrm{~T}\right)$ a proximity effect induced resistance minimum is observed. (ii) In the field range $B \simeq 0.4-2 \mathrm{~T}$ the resistance is approximately constant. (iii) For $B>3.1 \mathrm{~T}$ the whole sample is driven normal conducting. The inset shows the sample geometry: A mesoscopic $\mathrm{Au}$ wire $\left(\mathrm{Au}^{\mathrm{W}}\right.$, length $L \simeq 1 \mu \mathrm{m}$, width $W \simeq 180 \mathrm{~nm})$ is connected to a $\mathrm{Nb}$ contact $\left(\mathrm{Nb}^{\mathrm{C}}+\mathrm{Nb}^{\mathrm{R}}\right)$ and an $\mathrm{Au}$ contact $\left(\mathrm{Au}^{\mathrm{R}}+\mathrm{Au}^{\mathrm{C}}\right)$ (mesoscopic two-probe arrangement). (b) Conductance fluctuations of sample 1 in the NS-case $(0.4 \mathrm{~T}<B<1.9 \mathrm{~T}) \mathrm{rms}\left(G_{\mathrm{NS}}\right)=0.16 \pm 0.02 e^{2} / h$ and the $\mathrm{N}$-case $(3.1 \mathrm{~T}<B<9 \mathrm{~T}) \operatorname{rms}\left(G_{\mathrm{N}}\right)=0.058 \pm 0.003 e^{2} / h$.

The relative factor of eight results from (a) the fact that two elementary charges are driven through the system whenever an Andreev reflection occurs $(e \rightarrow 2 e)$ and (b) the presence of twice as many diffusionlike modes as in the N-regime. These additional modes are in many respects similar to the standard diffusive modes (cf. the discussion in Ref. (-4), that is their presence manifests in an additional factor of two (rather then in a structurally altered formula). We emphasize that in a normal metal, the scale $L_{\text {eff }}$ appearing in (11) represents the effective length of the sample, i.e., the length of the region where most of the voltage drops. In a NS-sample, however, $L_{\text {eff }}$ is twice that length. This is a consequence of the fact that both the incoming electrons and the outgoing holes have to traverse the system diffusively [5, 12.

We next discuss the absolute magnitude of the CF. Equation (11) contains most of the information about conductance fluctuations we need to know. In the limit of low temperatures and vanishing inelastic interactions $\left(T, \tau_{\varphi}^{-1} \rightarrow 0\right)$, Eq.(11) yields the universal values 


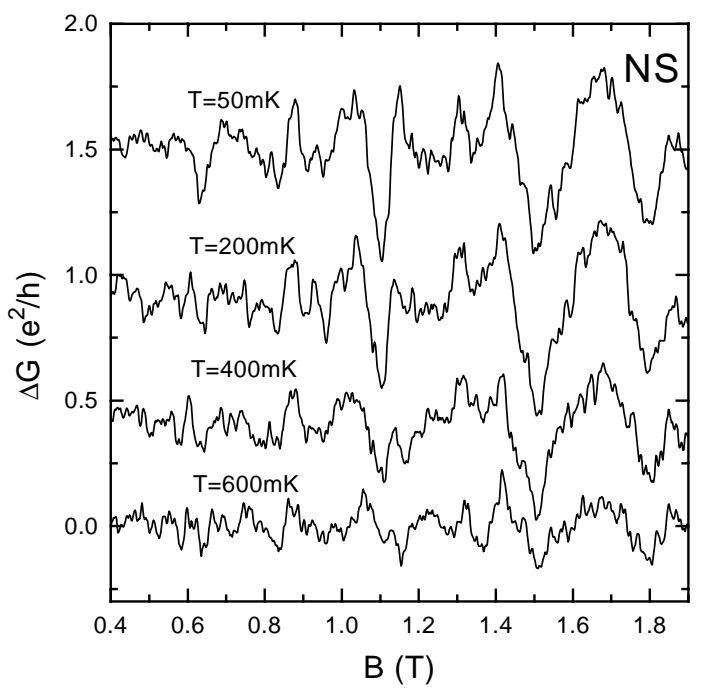

FIG. 2. NS-conductance fluctuations of sample 1 for different temperatures. For temperatures $T \geq 300 \mathrm{mK}$ the $\mathrm{CF}$ are reduced (cf. Fig.3). Traces are shifted for clarity.

$\operatorname{rms}\left(G_{\mathrm{N}}^{\text {theo }}\right)=0.258 e^{2} / h$ and $\operatorname{rms}\left(G_{\mathrm{NS}}^{\text {theo }}\right)=0.729 e^{2} / h$. In our experiment, however, the low temperature regime is characterized by the inequality $L_{T}>L_{\text {eff }} \gg L_{\varphi}$, where $L_{\varphi}=\left(D \tau_{\varphi}\right)^{1 / 2}$ and $L_{T}=\left(h D / k_{B} T\right)^{1 / 2}$ are the dephasing and thermal length, respectively. Under these circumstances the conductance fluctuations are reduced by a factor $\sim\left(L_{\varphi} / L_{\mathrm{eff}}\right)^{(4-d) / 2}$ below their universal, length independent value ( $d$ : dimensionality of the system). In order to interpret the experimental data we therefore carefully have to estimate the scale $L_{\text {eff }}$, i.e., the size of the sample region that contributes most to the resistance (or equivalently to the conductance fluctuations).

It is evident from the sample geometry shown in the inset of Fig. 11(a), that the main part of the voltage drop occurs in the narrow wire $\mathrm{Au}^{\mathrm{W}}$. The resistance of the wide contacts $\left(\mathrm{Nb}^{\mathrm{C}}+\mathrm{Nb}^{\mathrm{R}}\right.$ and $\mathrm{Au}^{\mathrm{R}}+\mathrm{Au}^{\mathrm{C}}$ in Fig. I] (a)) is about $1.2 R_{\square}$ each $\left(R_{\square}\right.$ : sheet resistance). The main voltage drop of a contact is located at the small rectangles $\mathrm{Nb}^{\mathrm{R}}$ and $\mathrm{Au}^{\mathrm{R}}$ corresponding to a resistance of about $0.5 R_{\square}$. The sheet resistances of the $\mathrm{Nb}$ and the $\mathrm{Au}$ differ by a factor of about four $\left(R_{\square}^{N b} \simeq 4 \Omega, R_{\square}^{A u} \simeq 0.9 \Omega\right)$. Thus the contribution of the $\mathrm{Au}$ rectangle $\mathrm{Au}^{\mathrm{R}}$ is about $0.5 \Omega$ which is less than $5 \%$ of the total resistance and neglected in the following. Hence, in the NS-case the voltage drop can be attributed to the wire $\mathrm{Au}^{\mathrm{W}}$. We obtain an effective length of $L_{\text {eff }}^{N S} \simeq 2 L \simeq 2 \mu \mathrm{m}$. In the case where the whole sample is normal conducting the situation is different. The $\mathrm{Nb}$ rectangle $\mathrm{Nb}^{\mathrm{R}}$ has a resistance of about $2 \Omega$ corresponding to $13 \%$ of the total resistance. The region contributing to the voltage drop is thus larger in the $\mathrm{N}$-case. Here, $L_{\text {eff }}$ is given by the regions $\mathrm{Au}^{\mathrm{W}}$ and $\mathrm{Nb}^{\mathrm{R}}$ resulting in $L_{\mathrm{eff}}^{\mathrm{N}} \simeq 1.8 \mu \mathrm{m}$. Of course this is only a rough estimate for the effective lengths of the samples. The main conclusion to be drawn is that the effective lengths $L_{\text {eff }}$ are roughly the same in both the $N$-case and the NS-case. To check the validity of the above consideration we have calculated the phase coherence length of the system via $L_{\varphi}=L_{\text {eff }}\left[\operatorname{rms}\left(G_{\text {exp }}\right) / \operatorname{rms}\left(G_{\text {theo }}\right)\right]^{2 / 3}$ using the previously estimated values of $L_{\text {eff }}$. The dephasing length $L_{\varphi}$ is expected to be roughly independent of the strength of the magnetic field (as long as the latter is sufficiently strong to break time reversal invariance). Indeed we obtain almost constant values $L_{\varphi} \simeq 660 \pm 60 \mathrm{~nm}$ for the different regimes and samples [24], thereby supporting the correctness of our estimates for $L_{\text {eff }}$. Furthermore the value for $L_{\varphi}$ is in good agreement with previous studies [19,22].

Since $L_{\text {eff }}^{\mathrm{N}} \simeq L_{\text {eff }}^{\mathrm{N}}$, we can directly compare the respective rms values of the $\mathrm{CF}$. We obtain a ratio of

$$
\frac{\operatorname{rms}\left(G_{\mathrm{NS}}\right)}{\operatorname{rms}\left(G_{\mathrm{N}}\right)}=2.8 \pm 0.4
$$

for both samples (see table II). This ratio is in agreement with the theoretical value of $2 \sqrt{2}$ 约.5]. Earlier theoretical work predicted an enhancement by only a factor two 12. In contrast, the experiment confirms the recent results of Altland and Zirnbauer [4] and Brouwer and Beenakker [5]. Equation (2) represents the central result of our experiment. In the next few paragraphs we report on further characteristics of the NS fluctuations, in particular on their temperature and voltage dependence.

Figure 2 shows NS-magnetofingerprints in the temperature range beween $50 \mathrm{mK}$ and $600 \mathrm{mK}$. Up to $T \simeq 300$ $\mathrm{mK}$ we observe the low temperature saturation value of $\operatorname{rms}\left(G_{\mathrm{NS}}\right)=0.16 \pm 0.02 e^{2} / h$, as shown in Fig. 3. For higher temperatures the $\mathrm{CF}$ are supressed and follow a $T^{-1 \pm 0.1}$ law. A similar behavior is observed for sample 2 (cf. Fig. 3). Here the reduction starts at a somewhat higher temperature $T \simeq 400 \mathrm{mK}$. The $\mathrm{N}-\mathrm{CF}$ were investigated for temperatures between $45 \mathrm{mK}$ and $7 \mathrm{~K}$ (cf. Fig. 3). The reduction of the $\mathrm{N}-\mathrm{CF}$ starts at $T \geq 400$ $\mathrm{mK}$ and follows a $T^{-0.50 \pm 0.05}$ law for both samples.

In general, $\mathrm{CF}$ are supressed as soon as either $L_{\varphi}(T)$ or $L_{T}$ become smaller than $L_{\text {eff }}$ (cf. Eq.11). For higher temperatures they show a weak power law behavior [2] $\operatorname{rms}[G(T)] \sim T^{-\alpha}$. The exponent depends on various system characteristics such as the ratio between the different length scales, and its dimensionality. However, we do not have a compelling argument for why $\alpha$ should depend on the presence or absence of superconductivity. In other words, a conclusive explanation of the different power-law behaviours observed in the experiment is lacking. Nevertheless, we would like to speculate on a mechanism that might be responsible for this effect: We observe that the rms amplitudes of the NS-CF and the N-CF saturate at low temperatures although $L_{\text {eff }}>L_{\varphi}$ in our samples. We therefore conclude that $L_{\varphi}$ must be almost temperature independent at low temperatures. If the temperature is raised, $L_{T}$ and $L_{\varphi}$ decrease and thereby the $\mathrm{CF}$. The point now is that the decay rate of the CF sensitively depends on the effective dimensionality of the sample - the 


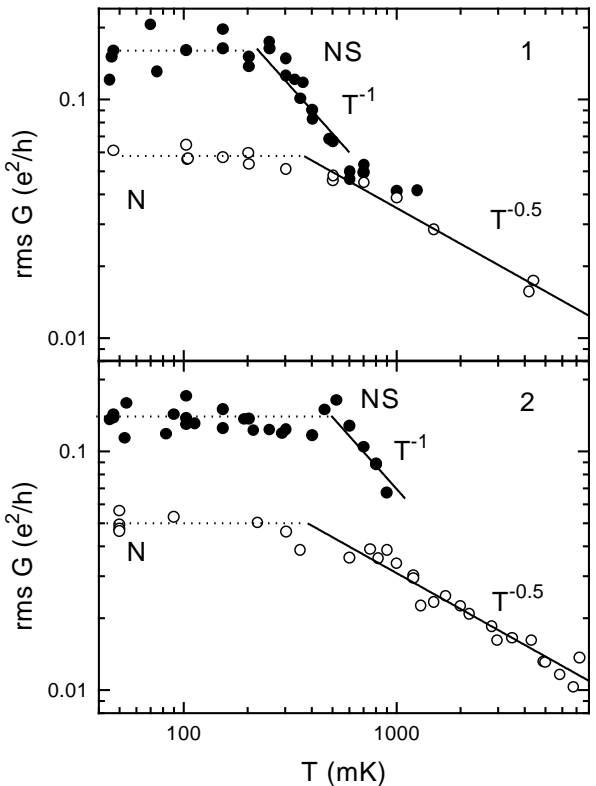

FIG. 3. rms amplitudes of the NS-CF $(\bullet)$ and the N-CF (o) for sample 1 (upper Fig.) and sample 2 (lower Fig.). The dotted lines show the low temperature saturation values (cf. tab. If). The reduction of $\operatorname{rms}\left[G_{\mathrm{NS}}(T)\right]$ starts at about $300-500$ $\mathrm{mK}$. The N-CF sets are reduced for $T \geq 400 \mathrm{mK}$. The solid lines correspond to $T^{-1}(\mathrm{NS})$ and $T^{-0.5}(\mathrm{~N})$ power laws.

lower the dimensionality, the higher the decay rate (cf. Ref. [2] for details). In regime (ii) our systems are clearly quasi-one-dimensional. However in (iii) the situation is different since the effectively two-dimensional wide $\mathrm{Nb}$ electrode contributes to the $\mathrm{CF}$. This might give rise to a less pronounced temperature dependence. Nonetheless it seems to be unlikely that a dimensional crossover of this kind can account for the whole effect.

Let us finally comment on the dependence of the $\mathrm{CF}$ on the measuring voltage. Voltages $V$ exceeding the value $V_{T h}=E_{T h} / e$ break the symmetry between particles and holes thereby leading to a destruction of the above mentioned additional diffusive modes. In our samples $V_{T h} \simeq 100 \mu \mathrm{V}$ 近, 5, 13, 14]. Indeed we do not observe any influence of $V_{a c}$ on size or temperature dependence of the NS-CF up to $V_{a c}=35 \mu \mathrm{V}$, which is of the same order as $V_{T h}$. For higher voltages $\operatorname{rms}\left(G_{\mathrm{NS}}\right)$ is reduced.

To summarize, we have measured the magnetoconductance fluctuations of mesoscopic $\mathrm{Au} / \mathrm{Nb}$ systems. By changing the magnetic field strength we induced a crossover from a normal-/superconducting to a purely normalconducting state. We found a relative enhancement factor $\operatorname{rms}\left(G_{\mathrm{NS}}\right) / \operatorname{rms}\left(G_{\mathrm{N}}\right)=2.8 \pm 0.4$ in good agreement with the theoretical prediction $\operatorname{rms}\left(G_{\mathrm{NS}}^{\text {theo }}\right) / \operatorname{rms}\left(G_{\mathrm{N}}^{\text {theo }}\right)=$ $2 \sqrt{2}[4$. For large temperatures the NS-CF behave like $\operatorname{rms}\left[G_{\mathrm{NS}}(T)\right] \sim 1 / T$ thereby showing a stronger temperature dependence than the N-CF.

We thank R. Gross, K. Jacobs, D.E. Khmelnitskii,
H. Micklitz, A. Nowack, and M. Zirnbauer for useful discussions. This work was supported by the Deutsche Forschungsgemeinschaft through SFB 341 and SFB 301 and by BMBF, grant 052KV134-(6) and 053KU234-(0).

[1] P.A. Lee and A.D. Stone, Phys. Rev. Lett. 55, 1622 (1985); B.L. Al'tshuler, Sov. Phys. JETP Lett. 12, 648 (1985).

[2] P. A. Lee, A. D. Stone, and H. Fukuyama, Phys. Rev. B 35, 1039 (1987).

[3] S. Washburn and R.A. Webb, Rep. Prog. Phys. 55, 1311 (1992), and references therein.

[4] A. Altland and M.R. Zirnbauer, Phys. Rev. B 55, 1142 (1997).

[5] P.W. Brouwer and C.W.J. Beenakker, Phys. Rev. B 52, 16772 (1995).

[6] A. Kastalsky et al., Phys. Rev. Lett. 67, 3026 (1991).

[7] V.T. Petrashov et al., Phys. Rev. Lett. 74, 5268 (1995).

[8] S.G. den Hartog et al., Phys. Rev. Lett. 76, 4592 (1996). S.G. den Hartog et al., Phys. Rev. Lett. 77, 4954 (1996).

[9] H. Courtois et al., Phys. Rev. Lett. 76, 130 (1996).

[10] S. Guéron et al., Phys. Rev. Lett. 77, 3025 (1996).

[11] P. Charlat et al., Phys. Rev. Lett. 77, 4950 (1996).

[12] Y. Takane and H. Ebisawa, J. Phys. Soc. Jpn. 61, 2858 (1992).

[13] I.K. Marmorkos, C.W.J. Beenakker, and R.A. Jalabert, Phys. Rev. B 48, 2811 (1993).

[14] P.W. Brouwer and C.W.J. Beenakker, Phys. Rev. B 52, R3868 (1995).

[15] Y.V. Nazarov and T.H. Stoof, Phys. Rev. Lett. 76, 823 (1996).

[16] A.F. Volkov, N. Allsopp, and C.J. Lambert, J. Phys.: Cond. Mat. 8, L45 (1996).

[17] A.F. Andreev, Sov. Phys. JETP 19, 1228 (1964).

[18] In zero magnetic field a reentrance of metallic conductivity occurs at low temperatures and voltages 11 . We observe a resistance minimum at $T \simeq 1 \mathrm{~K}$ in $R(T)$ and at $V_{d c} \simeq 100 \mu \mathrm{V}$ in IV-characteristics.

[19] K. Hecker et al. Phys. Rev. B 50, 18601 (1994).

[20] K. Fiegle, D. Diehl, and K. Jacobs, IEEE Trans. Appl. Supercond. 7, 3552 (1997).

[21] D.J. Thouless, Phys. Rev. Lett. 39, 1167 (1977).

[22] H. Hegger et al., Phys. Rev. Lett. 77, 3885 (1996).

[23] B.L. Altshuler, V.Kravtsov, and I.V. Lerner, Sov. Phys. JETP 64, 1352 (1986).

[24] As we do not observe a weak-localization contribution to the conductance the exact value of $L_{\varphi}$ cannot be evaluated in our samples. 